\title{
Prevalence and nutrient composition of menu offerings targeted to customers with dietary restrictions at US fast casual and full-service restaurants
}

\author{
Sophia V Hual,* ๑, Mark J Soto², Caroline G Dunn², Sara N Bleich ${ }^{2}$ and \\ Kelsey A Vercammen ${ }^{3}$ (] \\ 'Department of Nutrition, Harvard T.H. Chan School of Public Health, 677 Huntington Ave, Boston, MA 02 1 15, USA: \\ ${ }^{2}$ Department of Health Policy and Management, Harvard T.H. Chan School of Public Health, Boston, MA, USA: \\ ${ }^{3}$ Department of Epidemiology, Harvard T.H. Chan School of Public Health, Boston, MA, USA
}

Submitted 7 August 2020: Final revision received 2 December 2020: Accepted 5 January 2021: First published online 12 January 2021

\begin{abstract}
Objective: To examine the prevalence and nutrient composition of menu offerings targeted to customers with dietary restrictions at US fast casual and full-service chain restaurants.

Design: We used 2018 data from MenuStat, a database of nutrient information for menu items at large US chain restaurants. Five alternative diets were examined: gluten-free, low-calorie, low-carbohydrate, low-fat and vegetarian. Diet offerings were identified by searching MenuStat item descriptions and reviewing online menus. For each diet, we reported counts and proportions. We used bootstrapped multilevel models to examine differences in predicted mean kilojoules, saturated fat, $\mathrm{Na}$ and sugars between diet and non-diet menu items.

Setting: Forty-five US fast casual and full-service chain restaurants in 2018 (including 6419 items in initial analytic sample across small plates, salads and main dishes).

Participants: None.

Results: The most prevalent diets were gluten-free ( $n 631,9.8 \%$ of menu items), low-calorie ( $n$ 306, 4.8\%) and vegetarian ( $n$ 230, 3.6\%). Compared with non-diet counterparts, low-calorie main dishes had significantly lower levels of all nutrients examined and vegetarian main dishes had significantly lower levels of all nutrients except saturated fat. Gluten-free small plates had significantly fewer kilojoules, grams of saturated fat and milligrams of $\mathrm{Na}$ compared with non-diet small plates. Conclusions: A small proportion of fast casual and full-service restaurant menus are targeted towards customers with dietary restrictions. Compared with non-diet items, those classified as gluten-free, low-calorie or vegetarian generally have healthier nutrient profiles, but overall nutrient values are still too high for most menu items, regardless of dietary label.
\end{abstract}

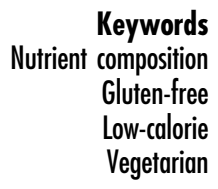

The weight loss market in the USA is worth more than $\$ 70$ billion annually and growing ${ }^{(1)}$. Between 2013 and 2016, about half of Americans reported attempting to lose weight in the past year, with over $60 \%$ of these individuals citing eating less as a method for weight loss ${ }^{(2)}$. In addition to popular commercial diets like Weight Watchers and Atkins, there has been a proliferation of other dietary approaches such as veganism, gluten-free, ketogenic, Paleolithic and intermittent fasting ${ }^{(3,4)}$ - all of which use strict guidelines to help consumers determine what or when to eat.
Although scientific consensus has yet to be reached on the long-term weight loss efficacy of these diets ${ }^{(5-7)}$, shortterm weight loss has been shown to be achievable for some ${ }^{(8-11)}$. In addition to weight loss, Americans are increasingly turning to alternative diets for reasons such as personal/moral preferences (e.g. a vegan diet out of concern for the environment) and accommodating food intolerances (e.g. a gluten-free diet to manage celiac disease).

In response to this rising demand, food manufacturers have started producing food and beverage products 
targeted to customers following restricted diets, including gluten-free (136\% increase between 2013 and 2015) ${ }^{(12)}$, plant-based (6.4\% increase between 2010 and 2015) ${ }^{(13)}$ and Paleolithic diets (35\% increase between 2015 and $2016)^{(14)}$. Despite the rise in popularity of these diets, there is little data on the presence or nutritional quality of these foods in the restaurant setting. This is an important gap in the literature given the major role that restaurants play in the USA, with Americans now spending more money dining out than on groceries ${ }^{(15)}$. Past analyses of chain restaurant menus as a whole have shown that, while the kilojoules in newly introduced items have consistently declined, nutritional changes over time have been minor and inconsistent across menu categories ${ }^{(16-18)}$. One recent study examined the nutrition composition of menu offerings at popular US restaurants, finding that offerings with claims related to health (e.g. 'nutritious'), product sourcing (e.g. 'local') and vegetal items (e.g. 'vegan') tended to align better with 2015-2020 Dietary Guidelines for Americans' recommendations for $\mathrm{Na}$, trans fat, saturated fat and fibre compared with items that did not have these claims ${ }^{(19)}$.

Our study will build off this prior research by examining menu items targeted to customers with dietary restrictions (gluten-free, low-calorie, low-carbohydrate (i.e. low-carb), low-fat and vegetarian). In addition to saturated fat and $\mathrm{Na}$, we will also examine the amounts of kilojoules and total sugar in these items - all of which are nutrients of public health interest. Given prior evidence that restaurant foods tend to be high in energy, fat and $\mathrm{Na}^{(20)}$, it is important to understand if restaurant items catered to those with dietary restrictions have similar, better or worse nutrient compositions than non-diet items. Specifically, this exploratory study will describe the proportion of menu items in US fast casual and full-service chain restaurants targeted at those with dietary restrictions and describe the nutrient composition of these items compared with non-diet menu items.

\section{Methods}

\section{Restaurant sample}

We used 2018 data from MenuStat, a database maintained by the New York City Department of Mental Health and Hygiene that catalogues nutrition information for a majority of the top 100 grossing chain restaurants in the USA ${ }^{(21)}$. Our study included 45 fast casual and full-service chain restaurants, categorised based on previously established objective criteria (see online supplementary material, Supplement A) ${ }^{(22)}$. We excluded fast food restaurants from these analyses as the focus was on restaurants that consumers perceive as healthier than fast food, even though entrées from fast casual and full-service chains tend to be higher in energy than entrées from fast food restaurants ${ }^{(23,24)}$. Fast casual restaurants were defined as those with at least two of the following four criteria: non-disposable utensils, onsite food preparation, no table service in establishments that have tables for seating and commitment to higher quality or fresh ingredients. Full-service restaurants were defined as those having table service.

\section{Menu items}

MenuStat categorises foods into the following menu categories: appetisers and sides, baked goods, beverages, burgers, desserts, entrées, fried potatoes, pizza, salads, sandwiches, soup, and toppings and ingredients. Baked goods, beverages and desserts were not included in this analysis to keep the focus on savoury food components, and toppings and ingredients were not included because they are not a well-defined category in MenuStat. Because of small sample sizes, we aggregated the remaining food groups into three major categories: (1) 'small plates' (including appetisers and sides, fried potatoes, and soup), (2) 'salads' (including salads) and (3) 'main dishes' (including burgers, entrées, pizza and sandwiches).

Items that were in the 'build your own' or 'you pick'style were excluded to prevent double counting what was offered at restaurants and to avoid measurement error involved in estimating nutrient ranges. Kids' menu items were also excluded because their nutrient profiles tend to differ from regular menu items, and there are considerably fewer items on the kids' menu than the regular menu. A total of 6419 items were included in the initial analytic sample (see online supplementary material, Supplement B).

\section{Alternative diets}

To identify which diets to include in this study, we conducted an exploratory search of all 45 online restaurant menus included in our sample for visual icons (e.g. ' $\mathrm{V}$ ' for vegan) and separate menu categories (e.g. 'Lighter Side') that indicated options for customers with dietary restrictions. We identified the five most frequently appearing alternative diets: gluten-free, low-calorie, low-carb, low-fat and vegetarian. During this review, we also identified keywords indicating an alternative diet that were unique to each restaurant (e.g. the 'under 600' menu label at Bonefish Grill; see online supplementary material, Supplement A). We then mapped individual menu items from all included restaurants onto the five alternative diets in several steps. First, we conducted an electronic search of MenuStat item descriptions using the identified keywords that may be indicative of a given diet. For example, item descriptions containing the word 'vegetarian' or 'vegan' were flagged as vegetarian (see online supplementary material, Supplement C). Then, one author (S.V.H.) hand-screened all items flagged in the electronic search process to ensure accuracy and recategorised items that did not match (e.g. 'Vegetarian pita tacos w/Blackened Salmon' was recategorised from a vegetarian option to a non-vegetarian option). Next, the author reviewed the online menus of all restaurants to capture additional 
alternative diet items that may not have been identified from their MenuStat item description but were present on a diet-specific menu section or flagged with a dietspecific logo. Finally, a second author (K.A.V.) checked that all items were coded appropriately; discrepancies were flagged and discussed until both coders reached a consensus. See Supplement D for details on the numbers of items during each step.

\section{Analyses}

First, we examined the absolute number and proportion of gluten-free, low-calorie, low-carb, low-fat and vegetarian items offered at the included restaurants among the initial analytic sample, overall and by menu category (small plates, salads and main dishes). For descriptive statistics, we included all items that either directly met the criteria for the included diets (e.g. 'Burger on gluten-free bun') or the item description indicated that they could be modified to meet the criteria (e.g. 'Choice of substituting gluten-free bun').

Next, multilevel models with random intercepts for restaurant and random effects for diet type at the restaurant level were used to examine the nutrient composition of each alternative diet compared with all other menu items. Random effects were included to allow the effect of each alternative diet on the nutrient values to vary at the restaurant level. For each alternative diet and menu category, we fit four models, one for each nutrient as the dependent variable (i.e. kilojoules, saturated fat, $\mathrm{Na}$ and total sugars). Items were only included in the nutrient analysis if they had complete data for that nutrient (kilocalories $n$ 5982; saturated fat $n$ 5946; Na $n$ 5943; sugar $n$ 5727). In these models, the covariates were alternative diet, menu category, an interaction term between alternative diet and menu category, and an indicator for whether or not the item was shareable (to account for large portion sizes meant for multiple people). Standard errors were bootstrapped with 1200 repetitions and adjusted for clustering at the restaurant level to account for correlated errors among items within restaurants. Bootstrapping was used to account for the non-normal distribution of the error terms for nutrients. Post-regression, we used the margins command in Stata to estimate the predicted differences in mean kilojoules, saturated fat, $\mathrm{Na}$ and total sugars for each alternative diet compared with all other items by menu category (e.g. difference in kilojoules between vegetarian small plates and non-vegetarian small plates). Those that did not directly meet dietary restriction criteria were considered non-diet items in this analysis because their nutrition information would not reflect the given diet (e.g. nutrient information for a burger on wheat bun would reflect the wheat bun, even if a gluten-free substitution was possible). Due to small sample sizes, low-carb ( $n$ 19) and low-fat ( $n$ 6) diets were not included in this nutrient analysis.

Significance was considered at $P<0 \cdot 05$. Analyses were conducted in 2019 and 2020 using Stata 16.

\section{Results}

Of the diets examined, the gluten-free diet comprised the largest proportion of menu offerings at fast casual and full-service chain menus, with $9.8 \%(n$ 631) of all menu items either identified as being gluten-friendly or could be modified to fit such a diet (Fig. 1). Low-calorie options made up $4.8 \%$ ( $n$ 306) of the menu offerings and vegetarian options made up $3.6 \%$ ( $n$ 230). Neither low-carb (0.3\%, $n 19)$ nor low-fat $(0 \cdot 1 \%, n 7)$ menu items were widely available. For each alternative diet, main dishes made up the biggest proportion offered.

Figure 2 shows the differences in mean nutrient values for alternative diet items compared with non-diet items (see online supplementary material, Supplement E for full table of differences in mean nutrient values with $95 \% \mathrm{CI}$ ). Lowcalorie items from all menu categories had fewer kilojoules than items not labelled as low-calorie, although this difference was not statistically significant for small dishes. Gluten-free small plates (-528.9 kJ [95\% CI: $-890 \cdot 4$, $-167 \cdot 4] ;-126 \cdot 4 \mathrm{kcal}[95 \% \mathrm{CI}:-212 \cdot 8,-40 \cdot 0])$ and vegetarian main dishes (-625.1 kJ [95\% CI: -1177.4, 74.9]; -149.4 kcal [95\% CI: $-281.4,-17.9]$ ) had significantly fewer kilojoules than their non-diet counterparts.

With respect to saturated fat (Fig. 2b), point estimates for almost all alternative diets and menu categories suggested alternative diet menu items were lower in saturated fat compared with non-diet items, although significant differences were only observed among low-calorie salads $(-4.3$ grams [95\% CI: $-7 \cdot 0,-1 \cdot 5])$, low-calorie main dishes $(-5.4$ grams $[95 \% \mathrm{CI}:-8 \cdot 0,-2 \cdot 7])$ and gluten-free small plates $(-2 \cdot 1$ grams [95\% CI: $-3 \cdot 8,-0 \cdot 5])$.

There were significant differences in $\mathrm{Na}$ content (Fig. 2c) among gluten-free (-291.5 mg [95\% CI: $-468 \cdot 1$, $-114.9])$, low-calorie (-378.5 mg [95\% CI: -573.5 , -183.4]) and vegetarian (-411.0 mg [-744.7, -77.3]) main dishes compared with regular main dishes, with similar results among salads, though those differences were not significant.

Findings for total sugar (Fig. 2d) were more mixed: while gluten-free $(-2 \cdot 3 \mathrm{~g}$ [95\% CI: $-5 \cdot 0,0 \cdot 4])$, low-calorie $(-2.9 \mathrm{~g}[95 \% \mathrm{CI}:-5 \cdot 3,-0.4])$ and vegetarian $(-3.0 \mathrm{~g}[95 \%$ CI: $-5.9,-0.04])$ main dishes typically had lower amounts of total sugar compared with non-diet counterparts, small plate diet items had higher amounts of sugar compared with non-diet items, with vegetarian small dishes having significantly more total sugar $(7 \cdot 7 \mathrm{~g}[95 \% \mathrm{CI}: 1 \cdot 7,13 \cdot 8])$ than non-vegetarian small dishes.

\section{Discussion}

This study examined the prevalence and nutrient composition of menu offerings targeted to customers with dietary restrictions at US fast casual and full-service chain restaurants. Our findings suggest that restaurants are meeting 


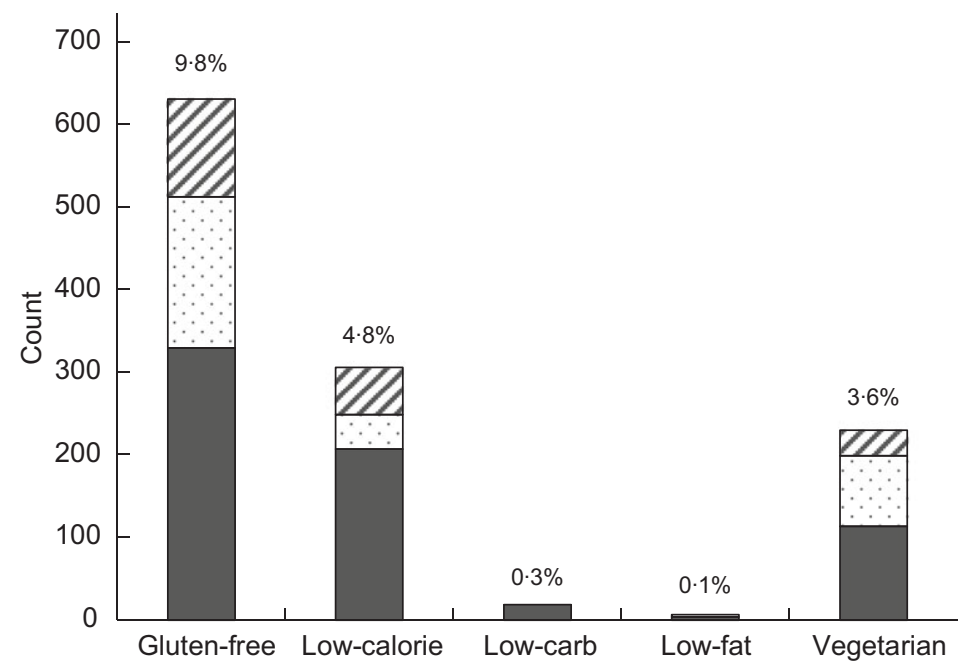

Fig. 1 Total count and percentage of items ${ }^{a, b, c}$ fitting alternative diet criteria in forty-five fast casual and full-service chain restaurant menus in the USA, 2018. ${ }^{\text {TT }}$ The main dish menu category includes the following MenuStat food categories: burgers, entrées, pizza and sandwiches. The small plates menu category includes the following MenuStat food categories: appetisers and sides, soup, and fried potatoes. ${ }^{b}$ Items that can be modified to fit the various diets were included in these counts (e.g. 'Choice of substituting gluten-free bun'). 'Percentages indicate percent of total menu items in initial analytic sample ( $N 6419)$ for each alternative diet. $\square$ Main dish; $\square$ small plates; r. salads

the consumer demand for alternative menu items by offering a variety of menu options meant for consumers with specific dietary needs. Our results provide evidence that alternative diet items have a modestly healthier nutrient profile compared with non-diet items, although there were some exceptions to this pattern and estimates were imprecise due to small sample sizes.

Our findings suggest that customers who are interested in identifying healthier menu items at fast casual and fullservice chain restaurants may benefit from ordering items that are offered for those with dietary restrictions, and that customers who follow alternative diets for another reason (e.g. moral or ethical) may benefit from the somewhat healthier profile of these items. In the context of sizable restaurant spending and the growing market for foods catered to consumers with dietary restrictions ${ }^{(12-15)}$, our findings are promising given that the recommended daily $\mathrm{Na}$ intake is $2300 \mathrm{mg}^{(25)}$, and items such as gluten-free small plates have nearly 500 fewer milligrams of $\mathrm{Na}$ - over one-fifth of the recommended amount - compared with all other small plates. Consuming dishes with less Na may have clinically beneficial health effects on outcomes such as hypertension $^{(26)}$. Furthermore, in light of evidence that replacing saturated fat in the diet with unsaturated fat lowers the risk of heart disease $\mathrm{e}^{(27,28)}$, it is of public health importance that almost all menu categories across the alternative diets examined had fewer grams of saturated fat than non-diet items.

However, such promising nutrient profile patterns are not necessarily replicated in the supermarket. For example, a recent study comparing gluten-free products to an equivalent food with gluten revealed a consistent pattern of higher saturated fat and $\mathrm{Na}$ in gluten-free products ${ }^{(29)}$, though these findings were not replicated in a study using a different list of foods or a review paper summarising results from several countries ${ }^{(30,31)}$. But while those studies examined packaged foods found at the supermarket, this study looked specifically at restaurant dishes, which tended towards lower levels of saturated fat and $\mathrm{Na}$. In regard to a vegetarian diet, studies have shown that those adhering to vegetarian lifestyles have better diet quality than nonvegetarians ${ }^{(32,33)}$, which is in line with our findings that foods labelled as vegetarian in the restaurant setting generally have lower levels of kilojoules, saturated fats, $\mathrm{Na}$ and sugar. While evidence exists showing that low-calorie diets are effective at weight loss, the means by which people restrict energy intake may determine the efficacy of such a weight loss method ${ }^{(34)}$. In our study, we see that lowcalorie main dishes were lower in all the nutrients examined when compared with all other main dishes, but restaurants outlined their own definitions for 'lowcalorie'. For instance, some specified 'low-calorie' as being less than 600 calories, while others had a 'under 700' section. This definition may be important to consider for people ordering off of the 'low-calorie' menu as a means for weight loss. Additionally, national calorie labelling at chain restaurants may help consumers identify lower energy items even if menus do not specifically have a 'low-calorie' section ${ }^{(35)}$.

We found that gluten-free items (or those that could be modified to be gluten-free) represented the largest proportion $(10 \%)$ of menu items, followed by low-calorie (5\%) and vegetarian ( $4 \%$ ) items. Low-carb and low-fat diet items accounted for a very small proportion of menu offerings ( $<0.5 \%$ combined). While the prevalence of celiac disease 
(a)

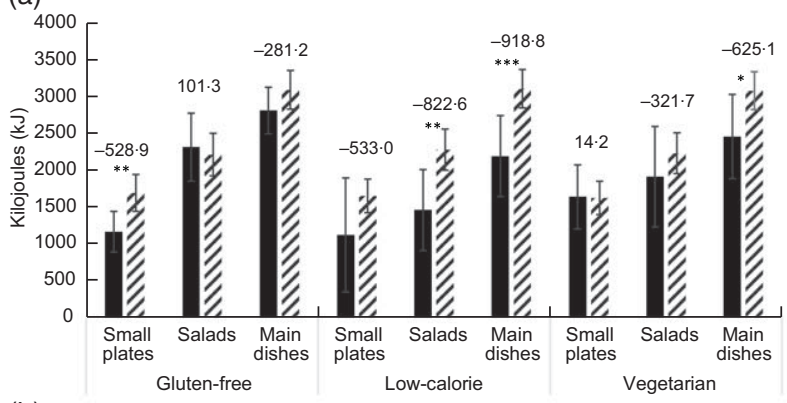

(b)

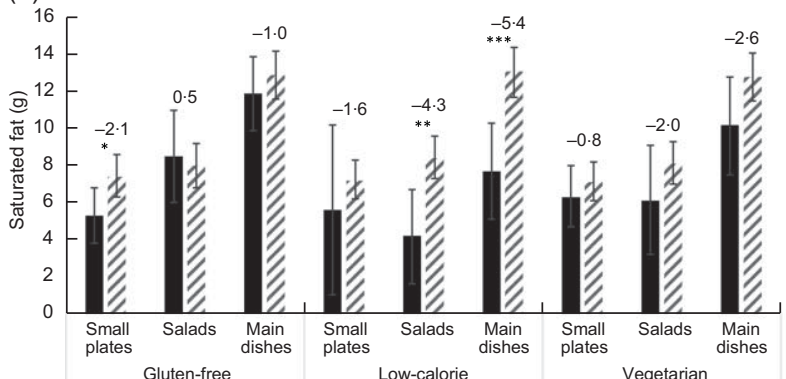

(c)

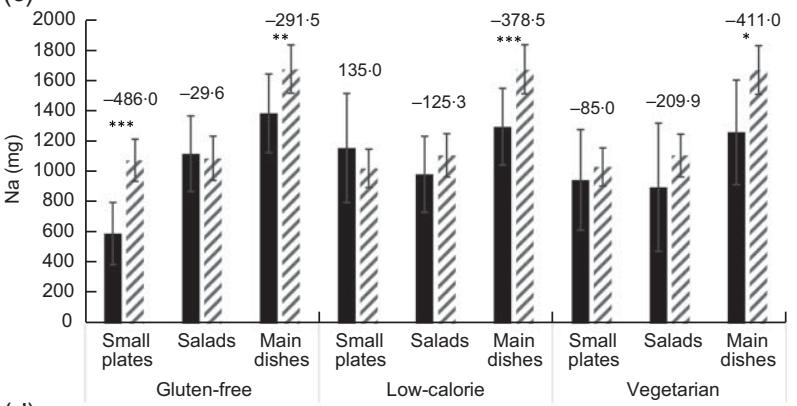

(d)

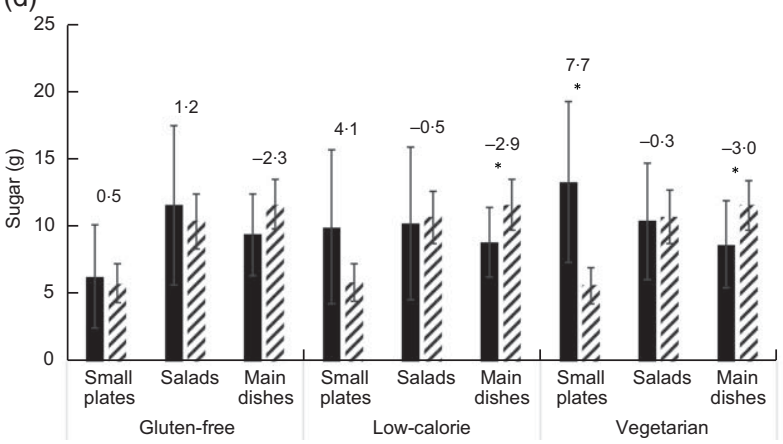

Fig. 2 Predicted differences in mean nutrient values ${ }^{\mathrm{a}}$ by alternative diet type ${ }^{b}$ and menu category ${ }^{c}$ in forty-five fast casual and full-service chain restaurants in the USA, 2018 $(1 \mathrm{kcal}=4.182 \mathrm{~kJ})$. ${ }^{\text {altems }}$ were excluded from nutrient analysis if they were missing that nutrient; total sample size by nutrient: kilocalories ( $n$ 5982), saturated fat ( $n$ 5946), $\mathrm{Na}(n 5943)$, sugar ( $n$ 5727). ' 'Non-diet' category/striped bars include all dishes within the menu category being examined that do not fit the specific alternative diet being examined. ${ }^{\mathrm{C} T h e}$ following MenuStat menu categories were excluded from analyses: beverages, baked goods, desserts, and toppings and ingredients. The Main Dish menu category includes the following MenuStat food categories: burgers, entrées, pizza and sandwiches. The Small Plates menu category includes the following MenuStat food categories: appetisers and sides, soup and fried potatoes. ${ }^{\star} P<0.05,{ }^{\star \star} P<0.01,{ }^{\star \star \star} P<0.001$. D Diet; ${ }^{*}$. non-diet in the USA ranges from less than 1 to $4.5 \%(36,37)$, an estimated $15-25 \%$ of consumers report seeking out gluten-free items for health and weight purposes, a demand that the restaurant industry has clearly responded to based on our findings ${ }^{(38)}$. The prevalence of those adhering to a vegetarian diet ranges from 2 to $5 \%$, which is aligned with the $3.6 \%$ of menu offerings in our sample ${ }^{(39-41)}$. Many Americans attempting to lose weight do so by eating less ${ }^{(2)}$, so it is notable that low-calorie items make up approximately $5 \%$ of fast casual and full-service chain menus. Finally, the low prevalence of low-fat and low-carb menu items corresponds to the shifting evidence base demonstrating that the quality of the fat and carbohydrate consumed has more health implications than the amount ${ }^{(42,43)}$. For instance, evidence suggests that substituting unsaturated fats for saturated fats or fibre-rich carbohydrates for simple carbohydrates both have health beneficial properties $^{(42,43)}$.

\section{Implications}

In general, fast casual and full-service chain restaurants offer meals that are high in energy and fat ${ }^{(23,24)}$. It is important for consumers to equip themselves with tools to make smarter choices while dining out, especially as Americans increasingly shift from eating in to eating out ${ }^{(15)}$. Consumers tend to inaccurately estimate the nutrient profile of restaurant foods ${ }^{(44)}$, but this study has demonstrated that in the context of fast casual and full-service chain restaurants in the USA, using labels meant for those with dietary restrictions may be a way to improve the relative healthfulness of food consumed outside the home. It is equally important to note, however, that the overall nutrient values are still too high for most menu items, regardless of dietary label. Research has shown that people perceive foods with labels such as 'gluten-free', 'vegetarian', 'low-fat' or 'low-carb' as generally healthier and/or lower in energy ${ }^{(45-48)}$, which is in line with the health halo effect of such labels ${ }^{(49)}$. But diners who order a small plate and main dish among alternative diet items can still consume up to $4184 \mathrm{~kJ}$ (1000 kcal) and over half of the daily recommended Na value in one meal, not including drinks and desserts. To promote lower energy choices, reformulation (rather than individual customer behaviour changes) is likely to have greater population reach and be more sustainable. While analyses have not yet examined the impact of the federal menu labelling mandate on changes in restaurant offerings, ample evidence suggests that restaurants have consistently reduced energies in newly introduced menu items for the past several years, perhaps in anticipation to the federal rule which was implemented in $2018^{(16-18)}$

As such, it remains vital that restaurants implement strategies to improve the healthfulness of foods, such as through portion size caps or changing the default to a 
healthier choice ${ }^{(50,51)}$. For instance, our study revealed that compared with items not labelled as low-calorie, lowcalorie main dishes were lower in all of the nutrients examined; thus, the default for restaurant main dishes might be to serve dishes low-calorie unless otherwise specified. However, restaurant owners may hesitate to offer lowcalorie dishes as the default because they may not be as palatable or profitable.

\section{Limitations}

First, because we relied on menus from 2019 to partially determine how foods were labelled, it was not possible to examine time trends in alternative diet offerings. Second, our data could not be normalised to a specific portion size (i.e. grams of saturated fat per 100 grams of food) as there was a high amount of missingness for the portion size variable in MenuStat. We adjusted for whether an item was shareable to account for very large portions but had to assume that if the items were not shareable, they were meant for one person. Third, MenuStat data include a limited number of restaurants and does not account for small, independent restaurants, so our results only speak to large US chains. Fourth, added sugars are not included in the MenuStat data, so we were unable to determine whether the higher amounts of sugar in vegetarian small plates were a result of natural or added sugars. Fifth, keywords were used to flag item descriptions that met diet criteria, so it may be possible that some keywords were overlooked. However, to capture as many relevant keywords as possible, all online menus were searched to see how items were labelled. Sixth, it is possible that some of the online menus did not present information on alternative diets that may have been present on the hard copies found in the restaurants; however, we did consistently find information about dietary restrictions on online menus. Seventh, not all included items had full nutrient information; as such, when conducting our nutrient analysis, we assumed that data were missing completely at random and conducted a complete case analysis. Finally, all flagged items were hand-searched to ensure correct categorisation into a type of alternative diet, so human error may have occurred. We attempted to circumvent major errors by having a double coder.

\section{Strengths}

This is the first study to explore both the prevalence and nutrient composition of menu offerings targeted to customers with dietary restrictions in US chain restaurants. We used a database systematically maintained by the New York City Department of Mental Health and Hygiene to run our analyses and supplemented the use of database descriptions with online menu reviews to ensure that we captured how restaurants are labelling their food items.

\section{Conclusion}

This study found that a small proportion of chain restaurant menus are targeted towards customers with dietary restrictions. Many of the nutrients examined for menu items that were gluten-free, low-calorie or vegetarian were consistently in a health beneficial direction, suggesting that consumers could potentially use these labels as a heuristic for relatively healthier restaurant choices whether or not they have dietary restrictions. However, continued surveillance of these differences going forward will be important to determine whether or not these and newly introduced offerings for those with dietary restrictions continue to be healthier than non-diet counterparts with respect to the public health nutrients of concern. Additionally, overall nutrient values are still too high for most menu items, regardless of dietary label.

\section{Acknowledgements}

Acknowledgements: None. Financial support: Sophia Hua is supported by the National Research Service Award (training grant T32 DK 007703) from the National Institutes of Health (NIH). Kelsey Vercammen was supported by a Canadian Institute of Health Research (CIHR) doctoral foreign study award (\#0492002603). This paper is solely the responsibility of the authors and does not necessarily represent the official views of the NIH or CIHR. Conflict of interest: None. Authorship: SVH developed the research question, conducted the statistical analysis, interpreted the data and drafted the manuscript. MJS provided statistical feedback, provided critical manuscript revisions and approved the final version of the manuscript. CDG interpreted the data, provided critical manuscript revisions and approved the final version of the manuscript. SNB developed the research question, provided critical manuscript revisions and approved the final version of the manuscript. KAV provided statistical feedback, interpreted the data, provided critical manuscript revisions and approved the final version of the manuscript. Ethics of human subject participation: Not applicable.

\section{Supplementary material}

For supplementary material accompanying this paper visit https://doi.org/10.1017/S1368980021000112

\section{References}

1. Marketdata LLC (2019) The U.S. Weight Loss \& Diet Control Market. Research and Markets. https://www. researchandmarkets.com/research/qm2gts/the_72_billion? $\mathrm{w}=4$ (accessed July 2020) 
2. Martin CB, Herrick KA, Sarafrazi N et al. (2018) Attempts to lose weight among adults in the United States, 2013-2016. NCHS Data Brief 313, 1-8.

3. Kim HS, Patel KG, Orosz E et al. (2016) Time trends in the prevalence of celiac disease and gluten-free diet in the US population: results from the National Health and Nutrition Examination Surveys 2009-2014. JAMA Intern Med 176, 1716-1717.

4. Nielsen (2018) Plant-Based Food Options Are Sprouting Growth for Retailers. New York: Nielsen Company.

5. Publishing HH (2015) The "Paleo Diet" - Back to the Stone Age? Boston, MA: Harvard Medical School.

6. Lebwohl B, Cao Y, Zong G et al. (2017) Long term gluten consumption in adults without celiac disease and risk of coronary heart disease: prospective cohort study. BMJ 357, j1892.

7. Obert J, Pearlman M, Obert L et al. (2017) Popular weight loss strategies: a review of four weight loss techniques. Curr Gastroenterol Rep 19, 61.

8. Howard BV, Manson JE, Stefanick ML et al. (2006) Low-fat dietary pattern and weight change over 7 years: the Women's Health Initiative Dietary Modification Trial. JAMA 295, 39-49.

9. Foster GD, Wyatt HR, Hill JO et al. (2010) Weight and metabolic outcomes after 2 years on a low-carbohydrate versus low-fat diet: a randomized trial. Ann Intern Med 153, $147-157$.

10. McManus K, Antinoro L \& Sacks F (2001) A randomized controlled trial of a moderate-fat, low-energy diet compared with a low fat, low-energy diet for weight loss in overweight adults. Int J Obes Relat Metab Disord 25, 1503-1511.

11. Sacks FM, Bray GA, Carey VJ et al. (2009) Comparison of weight-loss diets with different compositions of fat, protein, and carbohydrates. $N$ Engl J Med 360, 859-873.

12. Mintel (2015) Half of Americans think gluten-free diets are a fad while $25 \%$ eat gluten-free foods: Mintel. https://www. mintel.com/press-centre/food-and-drink/half-of-americansthink-gluten-free-diets-are-a-fad-while-25-eat-gluten-freefoods (accessed November 2019).

13. Reuters Plus (2019) Meat Alternatives Market-2019 Growth, Size, Share, Meat Substitute Industry, Cost-Structures, Trends, Protein-Base Type, Demand-Supply, Manufacturers Landscape, Outlook and Forecast-2024. https://www. reuters.com/brandfeatures/venture-capital/article?id=88471 (accessed November 2019).

14. Watson E (2017) Caveman eats: Paleo could hit \$4 bn by 2020 but it's never going to be Greek yogurt - IRI, Mintel. https:// www.foodnavigator-usa.com/Article/2017/07/10/Paleofoods-market-could-hit-4bn-by-2020-IRI-Mintel” ～(accessed July 2017).

15. Elitzak H \& Okrent A (2018) New U.S. Food Expenditure Estimates Find Food-Away-From-Home Spending Is Higher than Previous Estimates. Washington, DC: USDA Economic Research Service.

16. Wolfson JA, Moran AJ, Jarlenski MP et al. (2018) Trends in sodium content of menu items in large chain restaurants in the U.S. Am J Prev Med 54, 28-36.

17. Jarlenski MP, Wolfson JA \& Bleich SN (2016) Macronutrient composition of menu offerings in fast food restaurants in the U.S. Am J Prev Med 51, e91-97.

18. Bleich SN, Wolfson JA \& Jarlenski MP (2016) Calorie changes in large chain restaurants: declines in new menu items but room for improvement. Am J Prev Med 50, e1-e8.

19. Krobath D, Masters W \& Mueller M (2019) Menu descriptions and nutrient content of food items in U.S. restaurants (FSO201-19). Curr Dev Nutr 3, nzz051-FS02.

20. Auchincloss AH, Leonberg BL, Glanz K et al. (2014) Nutritional value of meals at full-service restaurant chains. J Nutr Educ Behav 46, 75-81.
21. Menustat (2018) MenuStat Methods \& Codebook. http:// menustat.org/Content/assets/pdfFile/MenuStat\%20Data\% 20Completeness\%20Documentation.pdf (accessed July 2019).

22. Bleich SN, Wolfson JA \& Jarlenski MP (2017) Calorie changes in large chain restaurants from 2008 to 2015. Prev Med 100, $112-116$

23. Scourboutakos MJ, Semnani-Azad Z \& L'Abbe MR (2013) Restaurant meals: almost a full day's worth of calories, fats, and sodium. JAMA Intern Med 173, 1373-1374.

24. Schoffman DE, Davidson CR, Hales SB et al. (2016) The fast-casual conundrum: fast-casual restaurant entrees are higher in calories than fast food. J Acad Nutr Diet 116, 1606-1612.

25. US Department of Health and Human Services \& US Department of Agriculture (2015) 2015-2020 Dietary Guidelines for Americans. https://health.gov/our-work/ food-nutrition/previous-dietary-guidelines/2015 (accessed December 2015).

26. Sacks FM, Svetkey LP, Vollmer WM et al. (2001) Effects on blood pressure of reduced dietary sodium and the Dietary Approaches to Stop Hypertension (DASH) diet. DASHSodium Collaborative Research Group. $N$ Engl J Med 344, 3-10.

27. Hu FB, Manson JE \& Willett WC (2001) Types of dietary fat and risk of coronary heart disease: a critical review. J Am Coll Nutr 20, 5-19.

28. Zong G, Li Y, Wanders AJ et al. (2016) Intake of individual saturated fatty acids and risk of coronary heart disease in US men and women: two prospective longitudinal cohort studies. BMJ 355, i5796.

29. Miranda J, Lasa A, Bustamante MA et al. (2014) Nutritional differences between a gluten-free diet and a diet containing equivalent products with gluten. Plant Foods Hum Nutr 69, 182-187.

30. Missbach B, Schwingshackl L, Billmann A et al. (2015) Gluten-free food database: the nutritional quality and cost of packaged gluten-free foods. PeerJ 3, e1337.

31. Melini V \& Melini F (2019) Gluten-free diet: gaps and needs for a healthier diet. Nutrients 11, 170.

32. Parker HW \& Vadiveloo MK (2019) Diet quality of vegetarian diets compared with nonvegetarian diets: a systematic review. Nutr Rev 77, 144-160.

33. Clarys P, Deliens T, Huybrechts I et al. (2014) Comparison of nutritional quality of the vegan, vegetarian, semivegetarian, pesco-vegetarian and omnivorous diet. Nutrients 6, 1318-1332.

34. Finer N (2001) Low-calorie diets and sustained weight loss. Obes Res 9, Suppl. 4, 290S-294S.

35. Long MW, Tobias DK, Cradock AL et al. (2015) Systematic review and meta-analysis of the impact of restaurant menu calorie labeling. Am J Public Health 105, e11-24.

36. Fasano A, Berti I, Gerarduzzi T et al. (2003) Prevalence of celiac disease in at-risk and not-at-risk groups in the United States: a large multicenter study. Arch Intern Med 163, 286-292.

37. Rubio-Tapia A, Ludvigsson JF, Brantner TL et al. (2012) The prevalence of celiac disease in the United States. Am J Gastroenterol 107, 1538-1544; quiz 1537, 1545.

38. Voiland A (2008) Gluten-free diet: a cure for some, a fad for most. 145, 66-68.

39. Kim H, Rotundo L, Song D et al. (2017) The prevalence and characteristics of vegetarian in the United States: a population-based study. Gastroenterology 152, S1016-S1016.

40. Cramer H, Kessler CS, Sundberg T et al. (2017) Characteristics of Americans choosing vegetarian and vegan diets for health reasons. J Nutr Educ Behav 49, 561-567 e561.

41. Newport F (2012) In U.S., 5\% Consider Themselves Vegetarians. Princeton, NJ: Gallup. 
42. Ludwig DS, Willett WC, Volek JS et al. (2018) Dietary fat: from foe to friend? Science 362, 764-770.

43. Reynolds A, Mann J, Cummings J et al. (2019) Carbohydrate quality and human health: a series of systematic reviews and meta-analyses. Lancet 393, 434-445.

44. Bates K, Burton S, Huggins K et al. (2011) Battling the bulge: menu board calorie legislation and its potential impact on meal repurchase intentions. J Consum Market 28, 104-113.

45. Besson T, Bouxom H \& Jaubert T (2019) Halo it's meat! the effect of the vegetarian label on calorie perception and food choices. Ecol Food Nutr 51, 1-18.

46. Ebneter DS, Latner JD \& Nigg CR (2013) Is less always more? The effects of low-fat labeling and caloric information on food intake, calorie estimates, taste preference, and health attributions. Appetite 68, 92-97.
47. Labiner-Wolfe J, Jordan Lin CT \& Verrill L (2010) Effect of low-carbohydrate claims on consumer perceptions about food products' healthfulness and helpfulness for weight management. J Nutr Educ Behav 42, 315-320.

48. Dunn CG, House L \& Shelnutt KP (2014) Consumer perceptions of gluten-free products and the healthfulness of glutenfree diets. I Nutr Educ Behav 46, S184-S185.

49. Williams P (2005) Consumer understanding and use of health claims for foods. Nutr Rev $\mathbf{6 3}, 256-264$.

50. Wootan MG (2012) Children's meals in restaurants: families need more help to make healthy choices. Child Obes $\mathbf{8}, 31-33$.

51. Cohen DA \& Story M (2014) Mitigating the health risks of dining out: the need for standardized portion sizes in restaurants. Am J Public Health 104, 586-590. 